\title{
Predictive modelling of cutting forces in end milling of titanium
}

\section{alloyTi6Al4V}

\author{
Yun Chen ${ }^{1,2}$, Huaizhong $\mathrm{Li}^{1,2^{*}}$, and Jun Wang ${ }^{2}$ \\ ${ }^{1}$ Griffith School of Engineering, Gold Coast campus, Griffith University, QLD 4222,
}

Australia
${ }^{2}$ School of Mechanical and Manufacturing Engineering, The University of New South Wales, Sydney, NSW 2052, Australia

\begin{abstract}
A cutting force model, based on a predictive model for orthogonal cutting, is developed for force predictions in end milling of titanium alloyTi6Al4V. The model assumes a semistationary process for the serrated chip formation. The Johnson-Cook material model that couples strain hardening, strain rate sensitivity and thermal softening effects is applied to represent the material strength. A thermal model considering the tool thermal properties is integrated to account for the high temperature rise due to the low thermal conductivity of Ti6Al4V. To extend the predictive model to milling, the end mill is discretised into several axial slices, and an equivalent cutting edge is used to include the end cutting edge effect
\end{abstract}

\footnotetext{
*Author to whom correspondence should be addressed: Dr Huaizhong Li, Griffith School of Engineering, Gold Coast campus, Griffith University, QLD 4222, Australia. E-mail: lihuaizhong@gmail.com; h.li@griffith.edu.au. Telephone: +61 (7) 5552 8252; Facsimile: +61 (7) 55528062
} 
caused by the first axial slice. The model is assessed by comparing its prediction with the experimental results and a mechanistic model for verification. The results show that the proposed model outperforms the mechanistic model with higher accuracy in force prediction.

Keywords: End milling; cutting forces; analytical modelling; titanium alloy; Ti6Al4V; machining; cutting.

\section{Nomenclature}

$a_{e} \quad$ Radial depth of cut in milling

$a_{p} \quad$ Axial depth of cut in milling

$A, B, C \quad$ Constants for the Johnson-Cook material model

$B(x) \quad$ Proportion of heat flowing into the tool at xposition along the interface

$\Psi_{r}, \Psi_{r}^{*} \quad$ Approach angle and modified approach angle

$C_{0} \quad$ Strain rate constant in the primary shear zone

$d_{w} \quad$ Width of cut in orthogonal cutting

$e_{n} \quad$ Static radial run-out for the $n$th tooth

$f_{t}, f_{r} \quad$ Feed per tooth and feed rate

$F_{c}, F_{t}, F_{r} \quad$ Cutting, thrust and lateral forces

$F_{s}, F_{f} \quad$ Shear and friction forces

$F_{x}, F_{y}, F_{z} \quad$ Forces in the $x, y$ and $z$ directions

$h_{1}, h_{2}, h_{1}^{*} \quad$ Uncut, cut chip thicknesses and modified uncut chip thicknesses

$k \quad$ Shear flow stress

$K_{c}, K_{t} \quad$ Thermal conductivities for the work material and tool

$l_{A B} \quad$ Length of the plane $A B$

$l_{\delta} \quad$ Tool-chip interface length

$m, n \quad$ Constants for the Johnson-Cook material model 


\begin{tabular}{|c|c|}
\hline$N_{f}$ & Normal force in the secondary shear zone \\
\hline$N_{t}$ & Number of teeth \\
\hline$P_{1}, P_{2}, P_{3}$ & Forces in the tangential, radial and axial directions \\
\hline$\dot{q}_{c}, \dot{q}_{t}$ & Heat generation rate for the chip and tool \\
\hline$R$ & Resultant force \\
\hline$R_{t}$ & Tool nominal radius \\
\hline$S$ & Workpiece specific heat \\
\hline$t$ & Time \\
\hline$T$ & Temperature \\
\hline$V, V_{s}, V_{c}$ & Cutting, shear and chip velocities \\
\hline$\varepsilon, \dot{\varepsilon}$ & Equivalent strain and strain rate \\
\hline$\phi_{n}$ & Normal shear angle \\
\hline$\gamma_{n}, \gamma_{n}^{*}$ & Normal rake angle and modified normal rake angle \\
\hline$\delta$ & Strain rate constant in the secondary shear zone \\
\hline$\Delta z, \Delta z^{*}$ & Elementary axial depth of cut and modified axial depth \\
\hline$\Delta \varphi$ & Rotation angle lag between two consecutive axial slices \\
\hline$\eta_{c h}$ & Chip flow angle \\
\hline$\varphi_{n, j}$ & Rotation angle for the $j$ th axial slice of the $n$th tooth \\
\hline$\varphi_{s}, \varphi_{e}$ & Enter and exit angles \\
\hline$\kappa_{r 1}$ & End cutting edge angle \\
\hline$\lambda_{s}, \lambda_{s}^{*}$ & Helix/inclination angle and modified helix/inclination angle \\
\hline$\mu_{n}$ & Normal friction angle \\
\hline$\rho$ & Workpiece density \\
\hline$\sigma$ & Normal stress \\
\hline$\tau$ & Shear stress \\
\hline$\Omega$ & Spindle rotation speed \\
\hline
\end{tabular}




\section{Introduction}

Titanium alloys have been widely used in the aerospace, power generation and biomedical industries, due to their excellent mechanical and chemical properties. However, these alloys are difficult to machine due to the low thermal conductivity, low elastic modulus, high rigidity, and strong affinity with tool materials. The high cutting temperature, chatter vibration and tool wear are the major issues which reduce the productivity in titanium alloy machining. Milling is a common material removal process for machining titanium alloys such as Ti6Al4Vin the manufacturing industry. To control and optimize the titanium alloy milling process, it is necessary to be able to predict the cutting forces. The knowledge of cutting forces is of importance for tool condition monitoring ${ }^{1}$ and chatter stability prediction/detection. ${ }^{2}, 3$ It has been used as an indicator for the cutting temperature estimation. $^{4}$

Mechanistic models are commonly used approaches for force prediction in the milling operations, in which the forces are evaluated using the force coefficients obtained from a series of cutting tests for a tool-workpiece couple with a given tool geometry. Kline and DeVor ${ }^{5}$ predicted the milling forces from average force coefficients which ignored the dependence of the force coefficients on the cutting conditions. Uriarte et al. ${ }^{6}$ introduced an average force coefficient model for force prediction in the micro end milling, with consideration of the effect of the tool deflection on the uncut chip thickness. Landers et al. ${ }^{7}$ applied a mechanistic force model with the average coefficients to select the optimal cutting conditions in milling thin titanium plates. To improve the model accuracy, instantaneous force coefficients are used instead of the average force coefficients for force prediction in the milling process. Yucesan and Altintas ${ }^{8}$ developed a three-dimensional (3D) mechanistic 
model for peripheral milling of a titanium alloy by taking into account the effects of the instantaneous chip thickness and rake angle on the force coefficients. Lamikiz et al. ${ }^{9}$ used the linear, quadratic and cubic polynomials to describe the dependence of the force coefficients on the axial position the ball-end mill. To apply the mechanistic model to the milling operation with any tool geometry, Budak et al. ${ }^{10}$ modeled the instantaneous force coefficients as functions of the shear angle, friction angle and shear yield stress using an orthogonal cutting database, and the instantaneous force coefficients and the oblique cutting analysis associated to the milling process were applied to force prediction in milling of Ti6Al4V. Despite the improvement developed by Budak et al., the predictive capability of the mechanistic models still depends on the empirical force coefficients determined for each toolworkpiece material pair. Large amounts of cutting tests are still needed, which is costly and inconvenient for tool designers and process planers.

Another approach for force predictions in machining of titanium alloys is the finite element (FE) method, which can include the thermo-mechanical effect on the simulated chip formation and cutting forces. Dhanorker and Ozel ${ }^{11}$ substituted the experimental tests using the virtual orthogonal cutting process simulated by the two-dimensional (2D) FE method to extract the cutting force coefficients. The thermo-mechanical effect on the cutting force coefficients was described using the Johnson-Cook material model. ${ }^{12}$ Gonzalo et al. ${ }^{13}$ used either the virtual orthogonal or oblique tests to extract the cutting force coefficients. Although the two methods provided the same precision for calculation of milling forces, the method based on the oblique tests costed much longer computation time than that based on the orthogonal tests. In addition to the extraction of the cutting force coefficients, Wang et al. ${ }^{14}$ used a hybrid model of 2D FE simulation and Oxley's theory ${ }^{15}$ to predict the cutting forces in 
milling Ti6Al4V. The 2D FE simulation was worked as a precursor to evaluate the constants in Oxley's theory. Although FE models can include complex thermo-mechanical effects, they are laboriousand are not easy to be directly applied to 3D machining operations. To extend FE methods to 3D machining operations, a series of 2D FE simulations are still required to evaluate the constants (e.g. cutting force coefficients) that are related to the machining process.

Analytical models are alternatives for force predictions. These models include the thermomechanical effect on the material strength, giving more insight into the physical mechanics in metal machining than the mechanistic models. Matsumura and Tamura ${ }^{16}$ employed an analytical model to investigate the effect of the material anisotropy on the cutting forces in milling of a titanium alloy. However, this model used several empirical equations that were derived from orthogonal cutting. Moufki et al. ${ }^{17}$ extended their thermo-mechanical oblique cutting model to milling of Ti6Al4V. This model simplified the chip formation as a narrow shear band only in the primary shear zone. Becze and Elbestawi ${ }^{18}$ applied an analytical model for force predictions in ball end milling of a hardened steel which is also well-known for the serrated chip formation. This model required extra orthogonal cutting tests and empirical parameters to estimate the strain and strain rate in the primary and secondary shear zones.Oxley's predictive machining theory is an analytical model that combines the effects of strain hardening, strain rate sensitivity and thermal softening. ${ }^{15} \mathrm{Li}$ and $\mathrm{Li}{ }^{19}$ extended Oxley’s predictive theory to helical end milling of medium carbon steel. Although Oxley's theory was developed for a steady state cutting process with continuous chips, it is still applicable to the serrated chip formation by assuming the serrated chip formation as a semi-stationary process according to Manyindo and Oxley's observations. ${ }^{20}$ Further, Vyas and Shaw ${ }^{21}$ suggested that 
the segmentation frequency was high up to $10-100 \mathrm{KHz}$ and a range of force fluctuation relative to the mean was $6 \%$, implying an insignificant variation in cutting forces due to chip segmentation.

It should be noted that Oxley's theory is independent on the tool material. However, the low thermal conductivity of titanium alloy Ti6Al4V causes the fact that most heat is dissipated from the tool. To account for the heat dissipation, the authors ${ }^{22}$ introduced an improved thermal model by including the tool thermal conductivity and incorporated the Johnson-Cook material model ${ }^{12}$ for the thermo-mechanical effects. This paper presents an extension of the authors' previous work for orthogonal cutting into milling operations. A 3D cutting force model is developed by considering the end cutting edge effect, and is verified under various cutting conditions in end milling of Ti6Al4V.

\section{Model development}

2.1 Modelling of orthogonal cutting process

The orthogonal cutting force model proposed by the authors ${ }^{22}$ is briefly introduced in this section. The basic assumption for the orthogonal cutting theory is that the serrated chip formation of titanium alloys is semi-stationary. Oxley's theory predicts the average values of the cyclic forces generated by the serrated chip formation. The chip formation for orthogonal

cutting is shown in Figure 1.The deformation is concentrated in two zones: a primary shear zone centred at the plane $A B$ and a secondary shear zone along the tool-chip interface. 


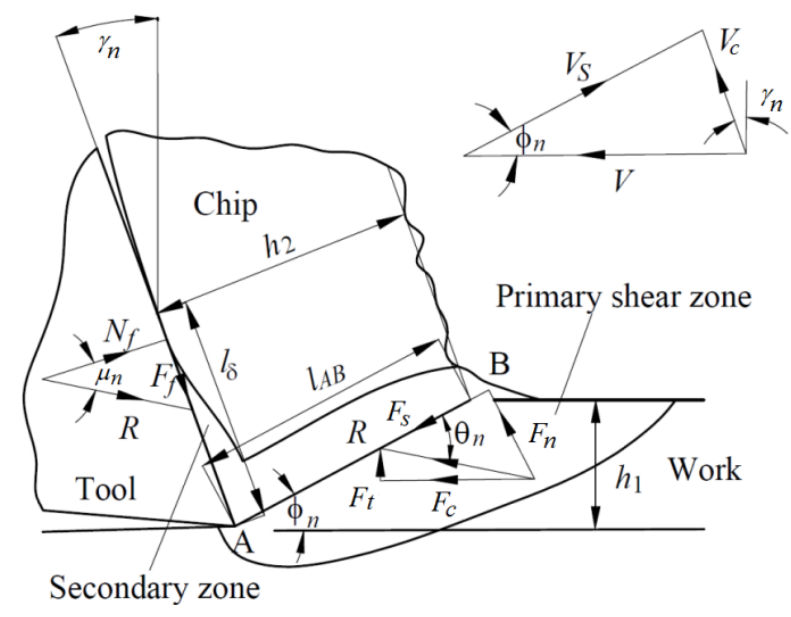

Figure 1.Chip formation in orthogonal cutting.

In the primary shear zone, the equivalent strain $\varepsilon_{A B}$ and strain rate $\dot{\varepsilon}_{A B}$ at the plane $A B$ are expressed as

$$
\varepsilon_{A B}=\frac{\cos \gamma_{n}}{2 \sqrt{3} \cos \left(\phi_{n}-\gamma_{n}\right) \sin \phi_{n}}, \dot{\varepsilon}_{A B}=C_{0} \frac{V_{s}}{\sqrt{3} l_{A B}}
$$

where $\gamma_{n}, \phi_{n}, l_{A B}$ and $V_{s}$ represent the rake angle, shear angle, the length of the plane $A B$ and the shear velocity, respectively, and $C_{0}$ is the strain rate constant at the plane $A B$. Adiabatic shear is assumed in the primary shear zone with no heat conducted into the work material and chip. Thus, the average temperature $T_{A B}$ at the plane $A B$ is defined as

$$
T_{A B}=F_{s} V_{s} \frac{1}{\rho S h_{1} V d_{w}}+T_{r}
$$

where $F_{S}$ is the shear force, $V$ the cutting speed, $d_{w}$ the width of cut, $S$ the workpiece specific heat, $\rho$ the workpiece density, $T_{r}$ the room temperature and $h_{1}$ is the uncut chip thickness. Using the Johnson-Cook material model, the equivalent shear stress $k_{A B}$ at the plane $A B$ becomes 


$$
k_{A B}=\frac{1}{\sqrt{3}}\left(A+B \varepsilon_{A B}{ }^{n}\right)\left(1+C \ln \left(\frac{\dot{\varepsilon}_{A B}}{\dot{\varepsilon}_{0}}\right)\right)\left(1-\left(\frac{T_{A B}-T_{r}}{T_{m}-T_{r}}\right)^{m}\right)
$$

where $A, B, C, m$ and $n$ are the Johnson-Cook material constants, and $\dot{\varepsilon}_{0}$ and $T_{m}$ are the reference strain rate and the melting temperature, respectively. The normal stress $\sigma_{N}$ 'at point $A$ is

$$
\sigma_{N}{ }^{\prime}=k_{A B}\left(1+\frac{\pi}{2}-2 \gamma_{n}-2 C_{0} \frac{B n \varepsilon_{A B}^{n}}{A+B \varepsilon_{A B}^{n}}\right)
$$

In the secondary shear zone, the shear flow stress $k_{\text {chip }}$ at the tool-chip interface is also evaluated using the Johnson-Cook material model

$$
k_{\text {chip }}=\frac{1}{\sqrt{3}}\left(A+B \varepsilon_{\text {int }}{ }^{n}\right)\left(1+C \ln \left(\frac{\dot{\varepsilon}_{\text {int }}}{\dot{\varepsilon}_{0}}\right)\right)\left(1-\left(\frac{T_{\text {int }}-T_{r}}{T_{m}-T_{r}}\right)^{m}\right)
$$

The equivalent shear strain $\varepsilon_{\text {int }}$ and strain rate $\dot{\varepsilon}_{\text {int }}$ at the tool-chip interface are defined

$$
\varepsilon_{\mathrm{int}}=\frac{l_{\delta}}{\sqrt{3} \delta h_{2}}, \quad \dot{\varepsilon}_{\mathrm{int}}=\frac{V_{c}}{\sqrt{3} \delta h_{2}}
$$

The average temperature $T_{\text {int }}$ at the tool-chip interface is derived from a $2 \mathrm{D}$ thermal model considering the tool thermal properties. The heat flow conducts in two directions in both the chip and tool, and there is also one-dimensional mass transfer along the chip flow direction in the chip. Accordingly, the heat balance equations for the chip and tool can be written as ${ }^{23}$ 


$$
\begin{gathered}
\frac{\partial^{2} T_{c}}{\partial x^{2}}+\frac{\partial^{2} T_{c}}{\partial y^{2}}+\frac{\dot{q}_{c}}{K_{c}}=\frac{\rho S}{K_{c}} \frac{\partial T_{c}}{\partial t}=\frac{\rho S}{K_{c}} V_{c} \frac{\partial T_{c}}{\partial x} \\
\frac{\partial^{2} T_{t}}{\partial r^{2}}+\frac{1}{r} \frac{\partial^{2} T_{t}}{\partial r}+\frac{1}{r^{2}} \frac{\dot{q}_{t}}{\partial \varphi^{2}}+\frac{\dot{q}_{t}}{K_{t}}=0
\end{gathered}
$$

Where $T_{c}, \dot{q}_{c}$ and $K_{c}$ are the temperature, heat generation rate and thermal conductivity for the chip, respectively, and $T_{t}, \dot{q}_{t}$ and $K_{t}$ for the tool. The heat generation rates $\dot{q}_{c}$ and $\dot{q}_{t}$ are assumed uniform along the tool-chip interface

$$
\dot{q}_{c}=\frac{(1-B(x)) F_{f} V_{c}}{l_{\delta} d_{w}}, \quad \dot{q}_{t}=\frac{B(x) F_{f} V_{c}}{l_{\delta} d_{w}} \text { if and only ifo } \leq x \leq l_{\delta}
$$

where $B(x)$ represents the proportion of the friction heat flowing into the tool at $x$ position along the tool-chip interface. The heat proportion $B(x)$ is solved using a finite difference method (Figure 2) to guarantee the equal temperatures at the interface on the tool side and the chip side. The average temperature $T_{\text {int }}$ is the mean of the temperatures for the discrete nodes along the tool-chip interface. 


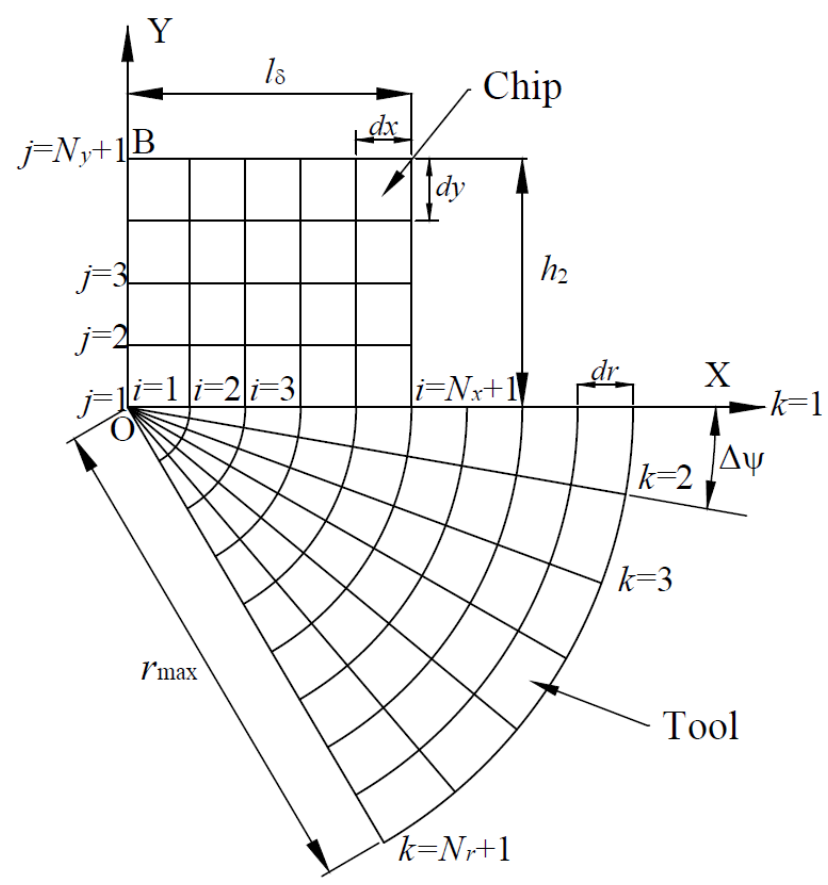

Figure 2. Chip and tool meshing for the finite difference method.

Assuming a uniform stress distribution over the tool-chip interface, the average shear stress $\tau_{\text {int }}$ and normal stress $\sigma_{N}$ at the interface are found from the equations

$$
\tau_{\text {int }}=\frac{F_{f}}{l_{\delta} d_{w}}, \sigma_{N}=\frac{N_{f}}{l_{\delta} d_{w}}
$$

The shear angle $\phi_{n}$ and the constant $C_{0}$ are determined from the stress equilibrium that $k_{\text {chip }}=\tau_{\text {int }}$ and $\sigma_{N}=\sigma_{N}{ }^{\prime}$. The last unknown $\delta$ is obtained from the minimum work principle ${ }^{15}$. Once the three unknown parameters $\phi_{n}, C_{0}$ and $\delta$ are determined, the cutting force $F_{c}$ and the thrust force $F_{t}$ are evaluated as 


$$
\begin{aligned}
& F_{c}=\frac{k_{A B} h_{1} d_{w}}{\sin \phi_{n} \cos \left(\mu_{n}+\phi_{n}-\gamma_{n}\right)} \cos \left(\mu_{n}-\gamma_{n}\right) \\
& F_{t}=\frac{k_{A B} h_{1} d_{w}}{\sin \phi_{n} \cos \left(\mu_{n}+\phi_{n}-\gamma_{n}\right)} \sin \left(\mu_{n}-\gamma_{n}\right)
\end{aligned}
$$

\subsection{Modelling of milling process}

The proposed force model from orthogonal cutting is extended to milling in this section. Figure 3shows a milling process with a helical end mill. The end mill with $N_{t}$ teeth rotates at a rotation speed $\Omega(\mathrm{rpm})$, and advances at a feed $f_{t}(\mathrm{~mm} /$ tooth $)$ or a feed rate $f_{r}(\mathrm{~m} / \mathrm{s})$. The end mill is discretised into several axial slices along its rotation axis in the $z$ direction. Each slice is equivalent to an oblique cutting process with an elementary axial depth of cut $\Delta z$ and an inclination angle $\lambda_{s}$. It is noted that a distinct difference exists between the first axial slice and the rest of the axial slices in their cutting actions as demonstrated by $\mathrm{Li}$ and $\mathrm{Li} .{ }^{19}$ The axial slices, except the first one, have only the peripheral cutting edges in cut. For the first slice, its end cutting edge, besides its peripheral cutting edge, is also involved in cut, which produces additional forces. When the axial depth of cut is small, the end cutting edge of the first slice considerably influences the chip flow angle and cutting forces. In order to allow for the multiple cutting edges, an equivalent edge that combines the peripheral and end cutting edges $^{24}$ is applied to evaluate the chip flow angle and cutting forces.

\section{Equivalent oblique cutting}

The equivalent oblique cutting for the axial slices except the first one is shown in Figure 4. The inclined cutting edge results in an additional force $F_{r}$ along the tool rotation axis in the $z$ direction. As the experimental results revealed an independent relationship between the 
inclination angle and the cutting forces $F_{c}$ and $F_{t}$, Lin et al. ${ }^{25}$ predicted the cutting forces $F_{c}$ and $F_{t}$ from the orthogonal theory by taking the inclination angle $\lambda_{s}$ equal to 0 irrespective of its true value with a normal rake angle $\gamma_{n}$. The additional force $F_{r}$ is determined by the fact that the resultant force should lie in the plane, normal to the tool rake face and containing the resultant frictional force acting in the chip flow direction

$$
F_{r}=\frac{F_{c}\left(\sin \lambda_{s}-\cos \lambda_{s} \sin \gamma_{n} \tan \eta_{c h}\right)-F_{t} \cos \gamma_{n} \tan \eta_{c h}}{\sin \lambda_{s} \sin \gamma_{n} \tan \eta_{c h}+\cos \lambda_{s}}
$$

The chip flow angle $\eta_{c h}$ is determined according to Stabler's flow rule which approximates the chip flow angle $\eta_{c h}$ equal to the inclination angle $\lambda_{s}{ }^{26}$

(a)

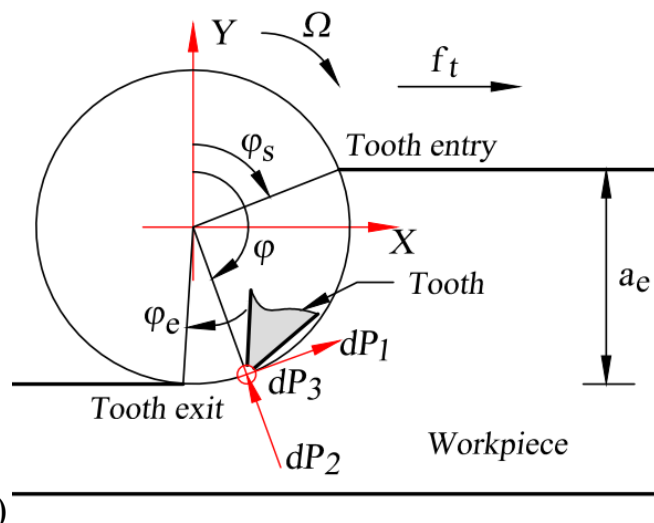

Figure 3. End milling process (a) discretisation of helical end mill (b) cross section of the end mill process. 


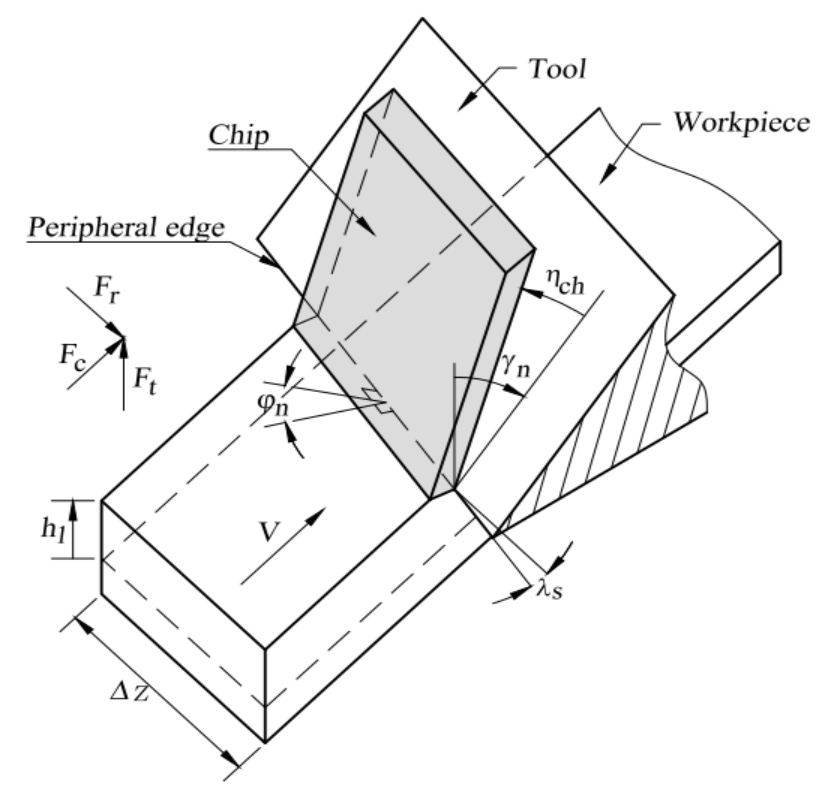

Figure 4. Equivelant oblique cutting for slices excluding the first one.

In order to allow for the end cutting edge effect for the first slice, the equivalent cutting edge developed by Hu et al. ${ }^{24}$ is applied. Figure 5shows the first axial slice presented in the toolin-hand coordinate system. ${ }^{27}$ The $X$-axis is along the cutting speed $V$, the $Y$-axis is along the direction of feed motion and the $Z$-axis is along the elementary axial depth of cut $\Delta z$. There are three basic planes $P_{s}, P_{s}^{\prime}$ and $P_{n}$ that define the tool geometry: $P_{s}$ contains the $X$-axis and the peripheral cutting edge $O B ; P_{s}^{\prime}$ is defined by the $X$-axis and the end cutting edge $O F ; P_{n}$ is perpendicular to $O B$. The approach angle $\Psi_{r}$ is made by the planes $P_{p}$ (plane $X Z$ ) and $P_{s}$. The end cutting edge angle $\kappa_{r 1}$ is the angle between the planes $P_{f}$ (plane $X Y$ ) and $P_{s}^{\prime}$. The normal rake angle $\gamma_{n}$ is measured in the plane $P_{n}$ between the rake face and plane $P_{r}$ (plane $Y Z$ ). The inclination angle $\lambda_{s}$ is the angle defined by lines $O D$ and $O D^{\prime}$ ( $D^{\prime}$ is the projection of the point $D$ in the plane $P_{r}$ ). 
The equivalent cutting edge $F D$ is such that the distance of point $F$ to the plane $P_{p}$ is the instantaneous feed or uncut chip thickness $h_{1}$ and the distance between point $D$ and the plane $P_{f}$ (plane $X Y$ ) is equal to $\Delta z$. Accordingly, the plane $P_{s}$ is modified as $P_{s}^{*}$ that contains the $X$-axis and $F D$, and the plane $P_{n}$ is referred to as $P_{n}^{*}$ perpendicular to $F D$. The rake, approach and inclination angles $\gamma_{n}^{*}, \Psi_{r}^{*}$ and $\lambda_{s}^{*}$ are redefined as

$$
\begin{gathered}
\gamma_{n}^{*}=\sin ^{-1}((\mathbf{L} 1 \times \mathbf{L} 2) \bullet \overline{\mathbf{F D}}) \\
\Psi_{s}^{*}=\sin ^{-1}((\mathbf{Z} \times \mathbf{L} 3) \bullet \mathbf{X}) \\
\lambda_{s}^{*}=\sin ^{-1}((\mathbf{L} 3 \times \overline{\mathbf{F D}}) \bullet \mathbf{L} 2)
\end{gathered}
$$

where $\overline{\mathbf{F D}}$ is the unit vector along the direction from point $F$ to point $D$. The unit vectors $\mathbf{L} 1$, $\mathbf{L} 2$ and $\mathbf{L} 3$ are along the lines of intersection between the plane $P_{n}^{*}$ and the rake face, planes $P_{n}^{*}$ and $P_{r}$, and planes $P_{s}^{*}$ and $P_{r}$, respectively. $\mathbf{L} 1, \mathbf{L} 2$ and $\mathbf{L} 3$ are evaluated as

$$
\mathbf{L} 1=\frac{\overline{\mathbf{F D}} \times \mathbf{P}_{\alpha}}{\left|\overline{\mathbf{F D}} \times \mathbf{P}_{\alpha}\right|}, \mathbf{L} 2=\frac{\overline{\mathbf{F D}} \times \mathbf{X}}{|\overline{\mathbf{F D}} \times \mathbf{X}|}, \mathbf{L} 3=\frac{\mathbf{X} \times \mathbf{L} 2}{|\mathbf{X} \times \mathbf{L} 2|}
$$

where $\mathbf{P}_{\alpha}$ is the unit vector normal to the tool rake face. 


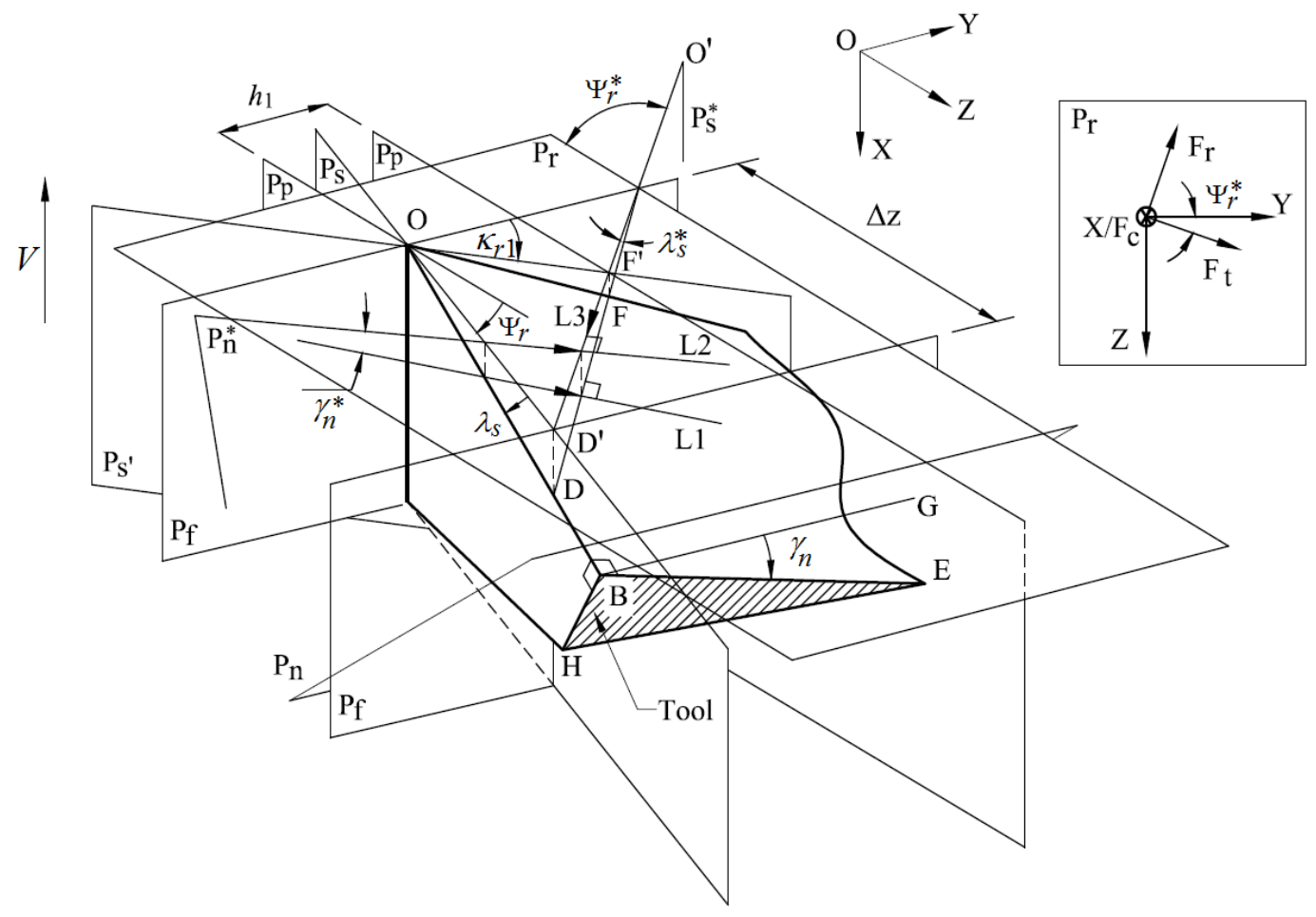

Figure 5.Equivalent cutting edge model for the first axial slice.

For the axial slices except the first one, the approach angle $\Psi_{r}$ is zero. However, the equivalent edge for the first axial slice may result in a nonzero equivalent approach angle $\Psi_{r}^{*}$, and therefore, $F_{t}$ and $F_{r}$ no longer act in the radial and axial directions, respectively (Figure 5). Instead, the elementary forces $d P_{1}, d P_{2}$ and $d P_{3}$ in the tangential, radial and axial directions (Figure 3(b)) are used to describe the force conditions on the tool

$$
\begin{aligned}
& d P_{1}=F_{c} \\
& d P_{2}=F_{t} \cos \Psi_{r}^{*}+F_{r} \sin \Psi_{r}^{*} \\
& d P_{3}=F_{t} \sin \Psi_{r}^{*}-F_{r} \cos \Psi_{r}^{*}
\end{aligned}
$$

It is noted that by applying the orthogonal theory for evaluations of $F_{c}$ and $F_{t}$ to the case that the approach angle is not zero, the uncut chip thickness and axial depth of cut should be modified as $h_{1}^{*}=h_{1} \cos \Psi_{r}^{*}$ and $\Delta z^{*}=\Delta z / \cos \Psi_{r}^{*}$. 


\section{Chip thickness model}

The uncut chip thickness is evaluated using a method proposed by Li et al. ${ }^{28}$ This method considers the trochoidal tooth trajectory generated by the rotational and translational motions of the tool, as well as the static radial run-out of teeth. The radial run-out is supposed to influence the entry angle $\varphi_{s}$ and exit angle $\varphi_{e}$ of flutes (Figure 3(b)). However, as the radial run-out is usually far smaller than the tool radius, such an effect on the entry and exist angles is usually negligible and is ignored in this study. The uncut chip thickness $|M N|$ at the time $t_{M}$ is shown Figure 6, where Point $M$ is the tool tip of the $j$ th axial slice at the current $n$th tooth, and point $N$ is the intersection point of the previous $(n+1)$ th tooth trajectory and the line $O M$ passing through the current tool tip and cutter axis. Points $O\left(x_{O}, y_{O}\right), M\left(x_{M}, y_{M}\right)$ and $N\left(x_{N}, y_{N}\right)$ are expressed respectively as

$$
\begin{aligned}
& x_{O}=f_{r} t_{M}, y_{O}=0 \\
& x_{M}=f_{r} t_{M}+\left(R_{t}+e_{n}\right) \sin \varphi_{n, j}, y_{M}=\left(R_{t}+e_{n}\right) \cos \varphi_{n, j} \\
& x_{N}=f_{r} t_{N}+\left(R_{t}+e_{n+1}\right) \sin \varphi_{n+1, j}, y_{N}=\left(R_{t}+e_{n+1}\right) \cos \varphi_{n+1, j}
\end{aligned}
$$

where $R_{t}$ is the nominal radius of the cutter, $e_{n}$ is the radial run-out of the previous $n$th tooth, and $t_{N}$ is the time at which the tool tip of the previous $(n+1)$ th tooth arrives at the point $N$. The rotation angle $\varphi_{n, j}$ for the current $n$th tooth at the $j$ th axial slice is defined as

$$
\varphi_{n, j}=\Omega t_{M}+\frac{2 \pi n}{N_{t}}-(j-1) \frac{\Delta z \tan \lambda_{s}}{R_{t}}
$$

Points $O, M$, and $N$ are in the same line, which gives

$$
\left(R_{t}+e_{n+1}\right) \sin \left(\Omega t_{N}-\Omega t_{M}+\frac{2 \pi}{N_{t}}\right)+f_{r}\left(t_{N}-t_{M}\right) \cos \varphi_{n, j}=0
$$

Equation (21) is solved using the Newton-Raphson method ${ }^{29}$ to determine the time $t_{N}$. Substituting $t_{N}$ into Equation (19), the uncut chip thickness is evaluated as 


$$
h_{1}=|M N|=|M O|-|N O|=\left(R_{t}+e_{n}\right)-\sqrt{\left(x_{O}-x_{N}\right)^{2}+\left(y_{O}-y_{N}\right)^{2}}
$$

The total milling forces are evaluated by summing the elementary forces contributed by the axial slices of all teeth(Figure 6)

$$
\begin{aligned}
& F_{x}=-\sum_{n} \sum_{j}\left(d P_{1} \cos \varphi_{n, j}+d P_{2} \sin \varphi_{n, j}\right) \\
& F_{y}=\sum_{n} \sum_{j}\left(d P_{1} \sin \varphi_{n, j}-d P_{2} \cos \varphi_{n, j}\right) \\
& F_{z}=\sum_{n} \sum_{j} d P_{3}
\end{aligned}
$$

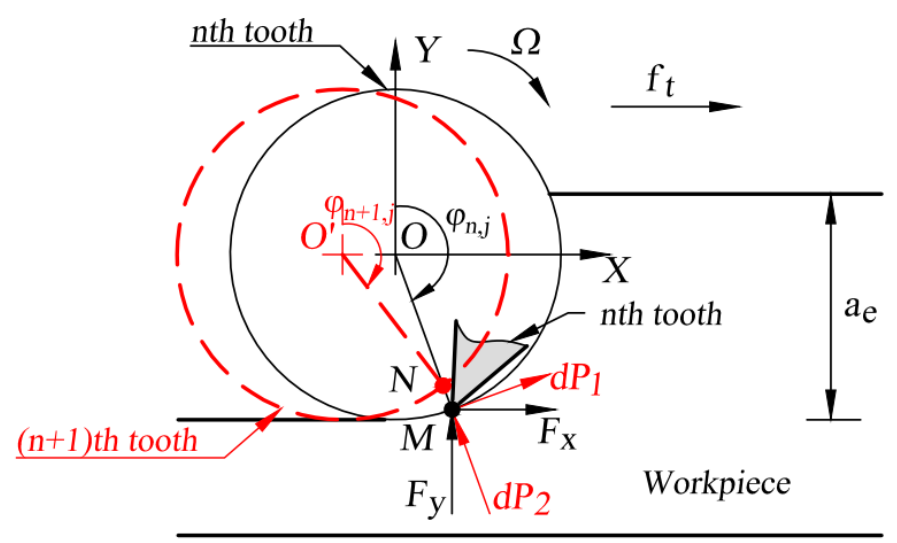

Figure 6. Trajectories of two consecutive teeth.

\section{Model verification}

Milling tests have been conducted on a Deckel Maho 5-axis CNC milling centre under dry cutting conditions. The titanium alloy Ti6Al4V workpiece was mounted on the top of a Kistler 9257B dynamometer which measured the cutting forces in three directions. The $x$ direction is the feed direction. The detailed workpiece properties can be referred to the

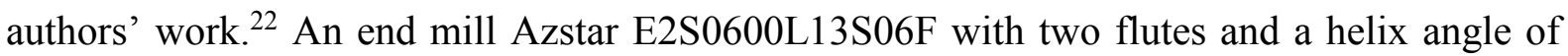
$30^{\circ}$ was used. The end mill has a nominal diameter of $6 \mathrm{~mm}$, and its measured normal radial 
rake angle $\gamma_{n}$ is $-4^{\text {o }}$. Table 1 lists the cutting conditions for 5 sample milling tests with a $25 \%$ radial immersion. The maximum tooth passing frequency of all the tests was only $159 \mathrm{~Hz}$. The Kistler 9257B dynamometer has a natural frequency of $2.3 \mathrm{kHz}$ in $\mathrm{X}$, Y axes and $3.5 \mathrm{kHz}$ in $\mathrm{Z}$ axis when mounted on flanges. With a workpiece mounted on the dynamometer, there was a decrease in the natural frequency of the system ${ }^{30,31}$. Even so, the natural frequency of the dynamometer was far above the maximum tooth passing frequency. Furthermore, a lowpass filter was applied to the sensor signal, hence resonance in dynamometer was avoided and precise measurement results for milling forces could be guaranteed.

Table 1. Cutting conditions of selected milling tests $\left(a_{e}=1.5 \mathrm{~mm}\right)$.

\begin{tabular}{lllll}
\hline Test \# & $a_{p}(\mathrm{~mm})$ & $f_{t}(\mathrm{~mm} /$ tooth $)$ & $\Omega(\mathrm{rpm})$ & Milling mode \\
\hline 1 & 1 & 0.08 & 3185 & Down \\
2 & 1 & 0.08 & 4777 & Down \\
3 & 2 & 0.08 & 3185 & Down \\
4 & 1 & 0.05 & 3185 & Down \\
5 & 1 & 0.08 & 3185 & Up \\
\hline
\end{tabular}

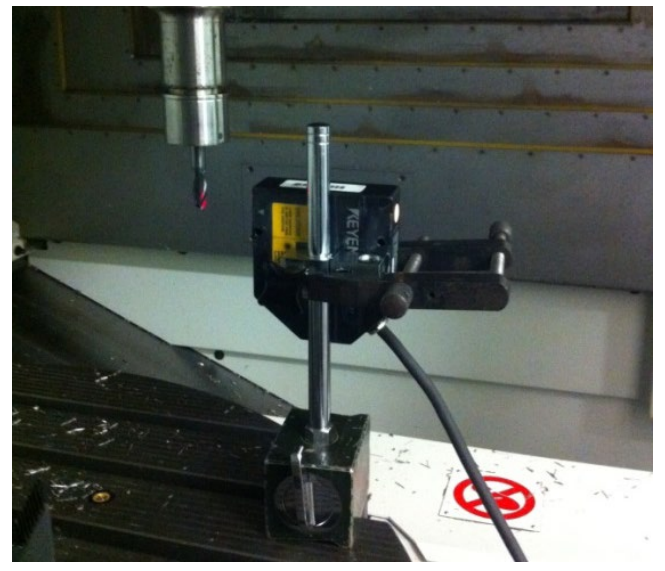

Figure 7. Radial run-out measurement.

The static radial run-out was measured after each cutter was clamped onto the spindle. A laser displacement sensor with a resolution of $0.01 \mu \mathrm{m}$ was used to measure the relative 
distance between the tool tip and the laser sensor as shown in Figure 7. Once the tool was rotated for one revolution, a trajectory of the tool tip relative to the laser sensor was recorded. A sample trajectory is shown in Figure 8. The relative distance between points 1 and 2 is the static radial run-out as defined by Jin and Altintas. ${ }^{32}$ Taking flute 2 as the reference flute with the nominal radius of $3 \mathrm{~mm}$, the radial run-out for flute 1 was $10 \mu \mathrm{m}$.

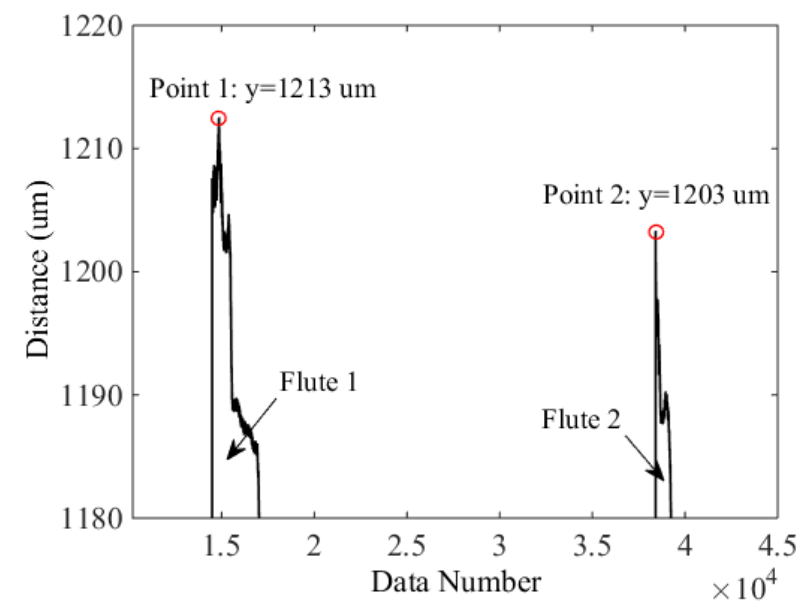

Figure 8.Trajectory of the tool tip relative to the laser sensor.

Although no chatter has been identified in all these tests, the measured forces still have some high frequency components around the natural frequency of the workpiece because of free vibrations during the no-cut portion, which distorts the force waveform. Therefore, a lowpass filter with a fixed cut-off frequency $800 \mathrm{~Hz}$ is applied to remove the high frequency components. The measured and predicted forces are compared in Figure 9. Although a lowpass filter has been applied to the measured forces, there are still continuous ripples on the measured forces. However, the predicted results are smooth as the vibration of the machine system is ignored. Figure 9(a)-(d) compare the measured and predicted forces from down 
milling tests at different cutting speeds, feeds and axial depths of cut. A good agreement between the measured and predicted results is observed. It can be seen that the cutting speed has marginal effect on the peak forces as revealed by both the measured and predicted forces. However, when the cutting speed increases (Tests 1 vs 2), the measured force waveforms show large-amplitude oscillations. A FFT spectral analysis of the measured forces reveals that this is caused by the larger magnitudes of high order harmonics of the tooth passing frequency for Test 2 than those for Test 1. In addition, a higher axial depth of cut or feed gives higher peak forces because of the increased chip load and thus the material removal rate.

The performance of the force model is also assessed for up milling as shown in Figure 9(e). The predicted peak forces are close to the measured values, but the phase width of the predicted force waveform differs from that of the experimental one, especially in the $y$ direction. Wan et al. ${ }^{33}$ attributed the difference in the phase width to the forces caused by the end cutting edge. These forces exist even when the peripheral cutting edge is not in cut, which pushes the effective cutting upper boundary beyond the exit angle $\varphi_{e}$. However, such an expansion of the effective cutting range is not considered in the current model. In addition, the radial run-out, as aforementioned, is also expected to influence the entry angle $\varphi_{s}$ and exit angle $\varphi_{e}$. Considering the radial run-out effect on the cutting range in up milling, the exit angle $\varphi_{e}$ for the $n$th tooth as defined by Schmitz and Smith ${ }^{34}$ should be modified as

$$
\varphi_{e}=\cos ^{-1}\left(\frac{R_{t}-a_{e}}{R_{t}+e_{n}}\right)
$$

where $a_{e}$ is the radial depth of cut. Equation (22) indicates that the exit angle $\varphi_{e}$ may vary for different teeth due to the radial run-out $e_{n}$, and a positive $e_{n}$ will increase the angle $\varphi_{e}$. 
Further, the force waveforms for Test 5 show larger oscillations than its corresponding waveforms in the down milling test (Test 1), revealing a stronger vibration in the up milling test. The strong vibration may also expand the effective cutting range.
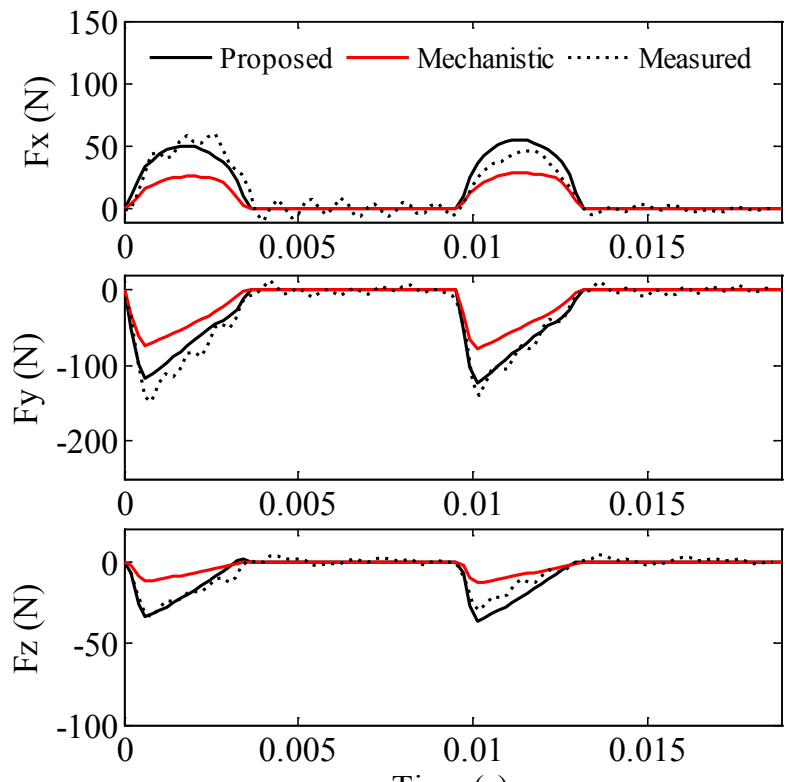

(a)

Time (s)
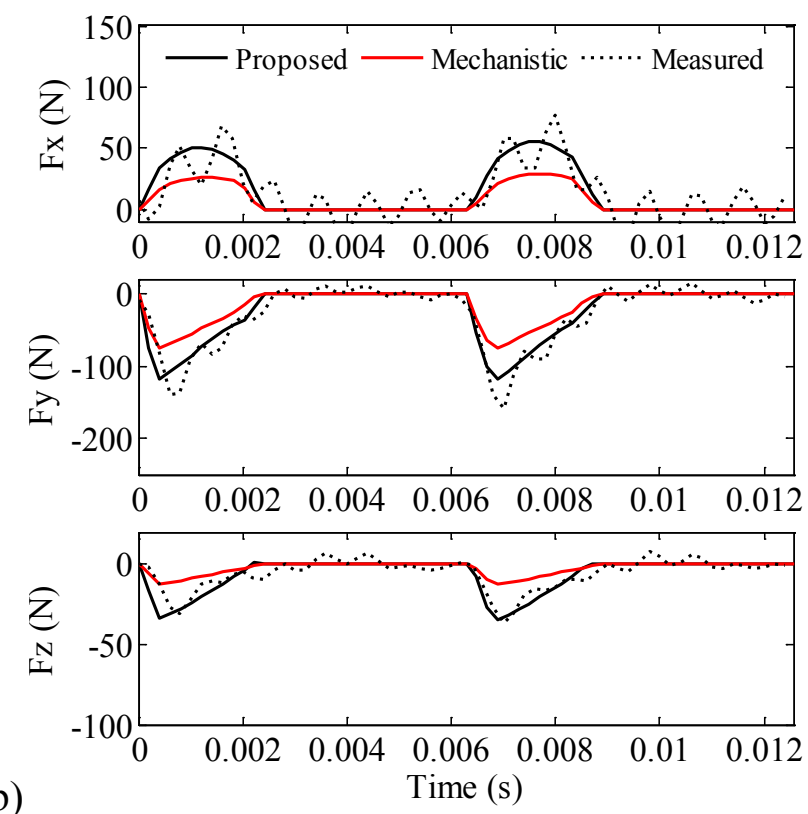

(b) 

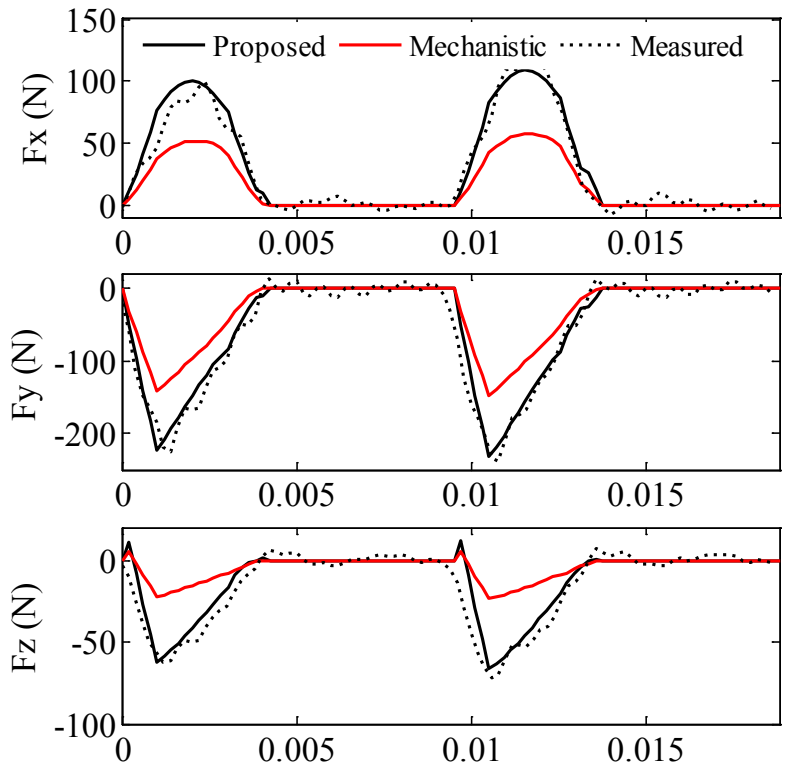

(c)

Time (s)
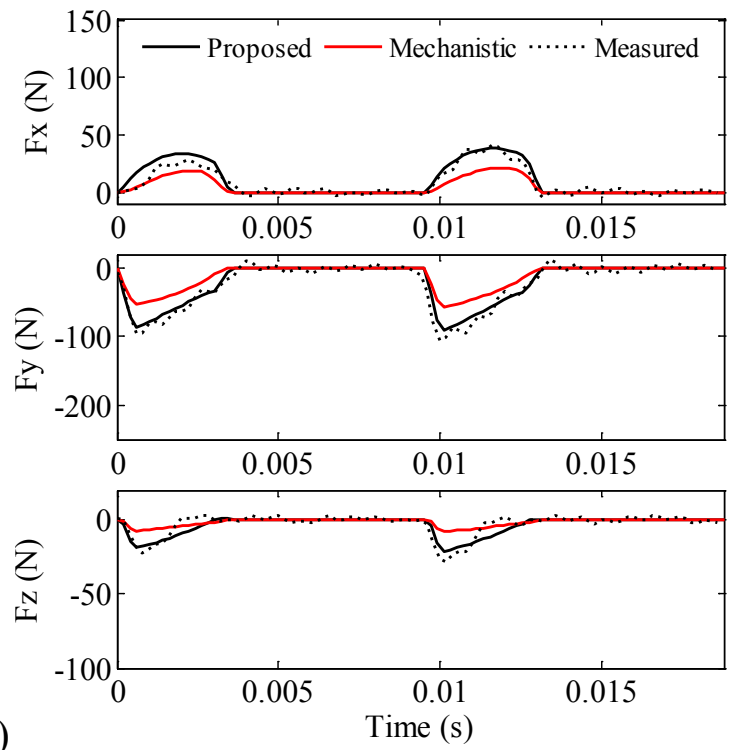

(d) 

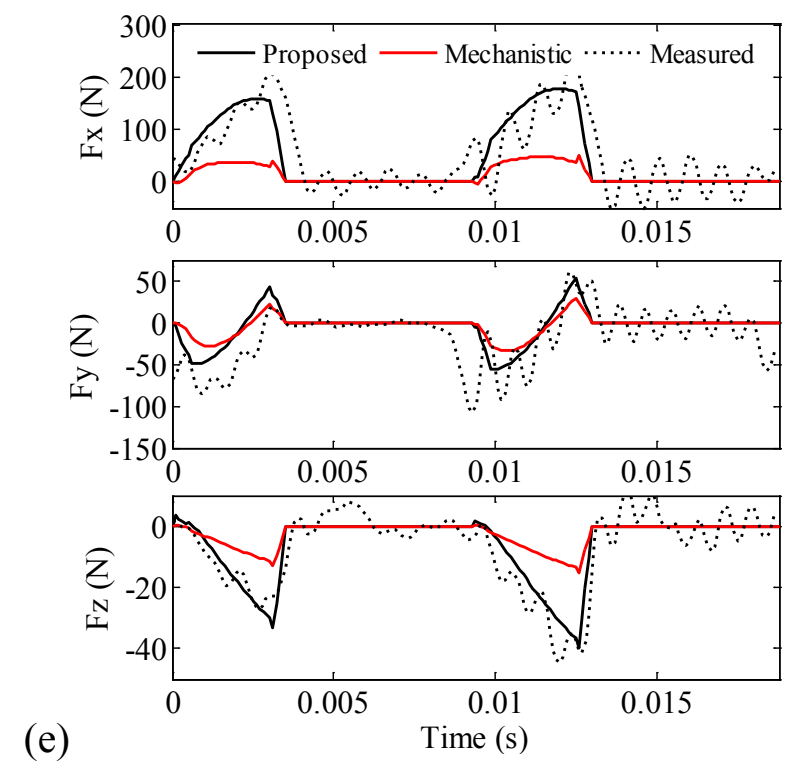

Figure 9. Comparison of predicted and measured forces (a) Test 1 (b) Test 2 (c) Test 3 (d)

Test 4 (e) Test 5. Cutting conditions are listed in Table 1.

In Figure 9, the force prediction using a mechanistic model as in Wan et al. ${ }^{35}$ is also presented for comparison. The force coefficients in the mechanistic model were identified from the average measured forces over one tooth passing period. It can be seen that the results from the mechanistic model show significant deviations from the measured values for all the investigated tests. The deviations are possibly caused by an inaccurate calibration of the force coefficients. Additionally, the mechanistic model requires matching the experimental forces and the instantaneous average chip thickness. As the measured forces are usually influenced by the tool-workpiece vibration, it is difficult to exactly identify the critical point at which a tooth begins cutting engagement. This also challenges the accurate calibration of the coefficients. On the other hand, the proposed method demonstrated more accurate force prediction results than the mechanistic model. It is actually built on an analytical force model that couples the thermal and mechanical effects, and is independent of the experimental data. Machining process models are used by the industrial practitioners to 
select optimum machining parameters. When the process models are not accurate enough, more conservative machining parameters have to be chosen and hence lead to loss of productivity and accuracy. Improvement of model accuracy can help to achieve optimum machining parameters such as feed, cutting speed, depth of cut, and cutting fluids. Even small changes in any of these parameters may have a significant effect on the surface quality and productivity. Therefore, it is important for the researchers to develop more accurate models.

Figures 10 and 11 present comparisons of the predicted and measured peak forces in the three principle directions under different cutting conditions, in the down and up milling operations, respectively. Two sets of force models were used in the force prediction, using the approaches with or without the equivalent cutting edge considered, respectively. The percentage errors of the simulated peak forces in Figures 10 and 11 by using the model with the equivalent cutting edge considered are less than $20 \%$, which indicates acceptable simulation accuracy. It can be seen that an increase of the feed per tooth caused a clear increment of the peak forces in all directions. The developed models are able to predict such a trend along with the increase of the feed. Both models with and without the equivalent cutting edge can predict the peak forces much closer to the measured results in the $z$ direction. It is found that the predicted peak forces $F_{x}$ and $F_{y}$ using the model with the equivalent cutting edge considered are closer to the experimental results than that without such a cutting edge considered, although both models underestimated the peak forces when the feed per tooth was high. One possible reason for the obvious deviation of the simulated results at high feed is that the tool-workpiece vibration is significant when the chip load is high, as can be seen in Figure 9(b) and (e). Although a low-pass filter was applied to the force signal, the cutoff frequency was still high and the vibration ripples still existed, which contributed to high 
measured peak force values. However, the influence of the cutting dynamics has not been included in the developed cutting force model. To further improve the model accuracy, the dynamic cutting process and tool-workpiece vibration should be taken into consideration in the cutting force modelling.
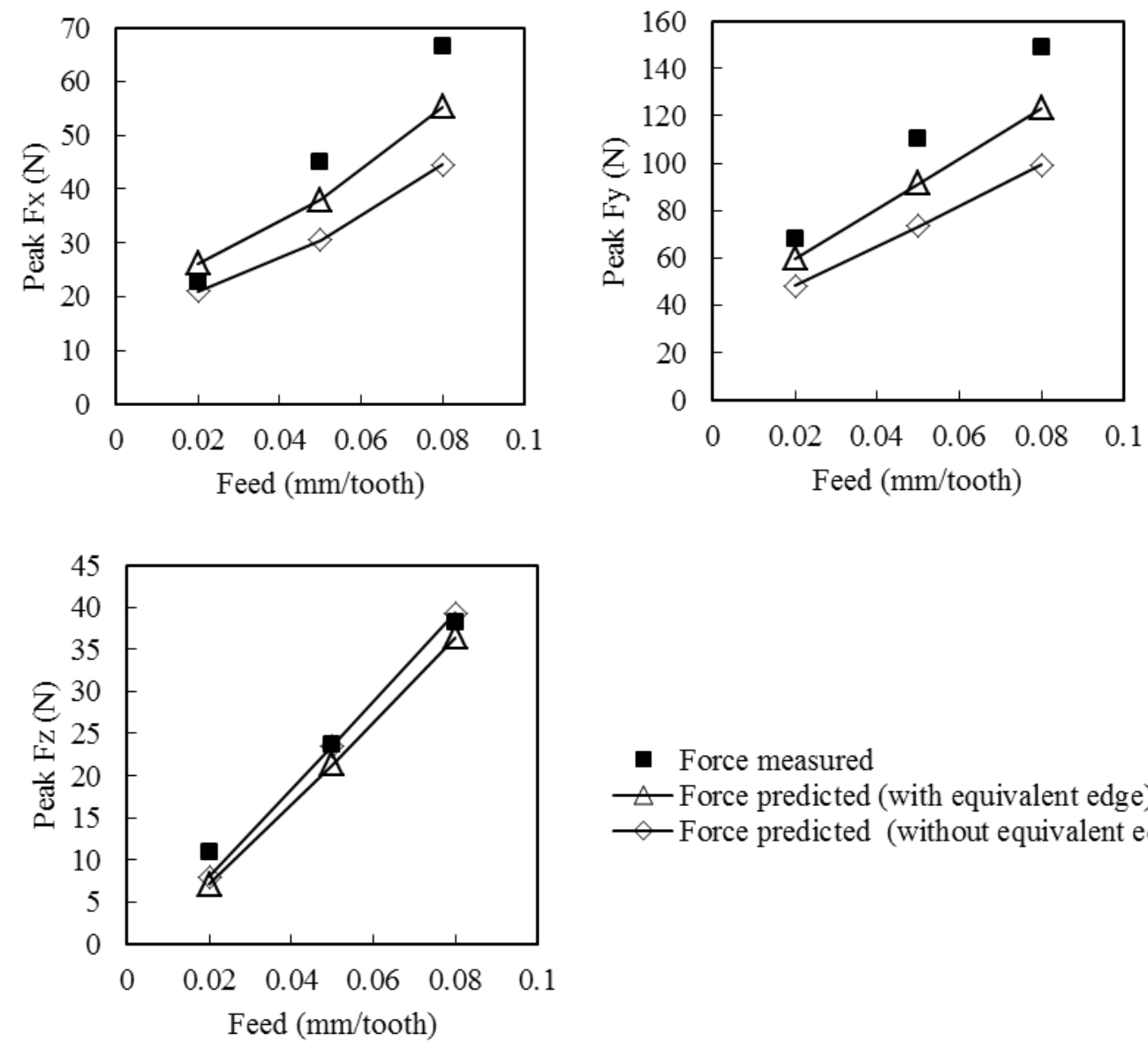

- Force measured

$\triangle$ Force predicted (with equivalent edge)

$\smile$ Force predicted (without equivalent edge)

Figure 10. Peak forces at $\Omega=3185 \mathrm{rpm}, a_{p}=1 \mathrm{~mm}$ and $a_{e}=1.5 \mathrm{~mm}$ (down milling). 

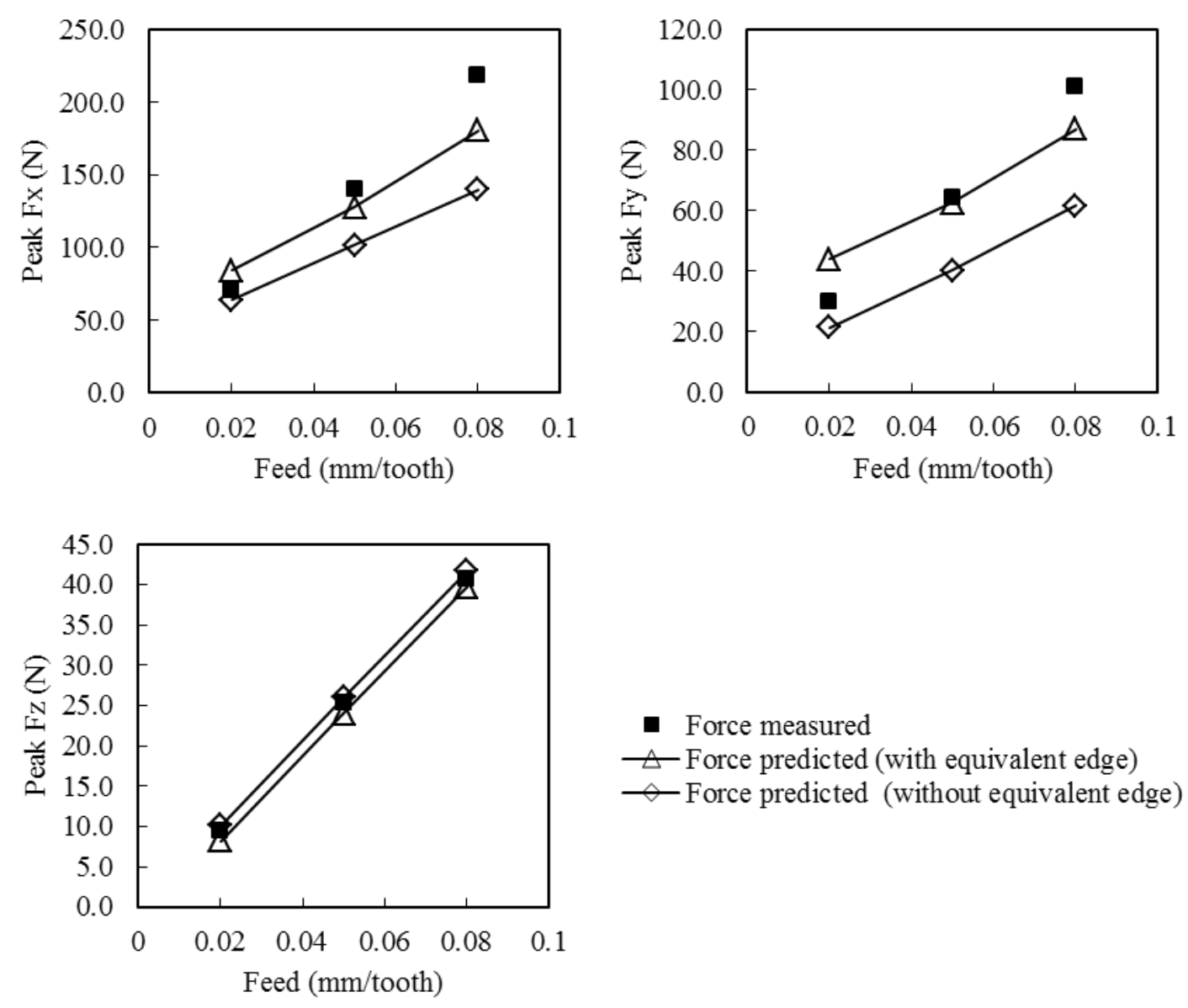

- Force measured

$\triangle$ Force predicted (with equivalent edge)

$\smile$ Force predicted (without equivalent edge)

Figure 11.Peak forces at $\Omega=3185 \mathrm{rpm}, a_{p}=1 \mathrm{~mm}$ and $a_{e}=1.5 \mathrm{~mm}$ (up milling).

\section{Conclusions}

In this paper, a predictive cutting force model in end milling of titanium alloy Ti6A14V has been developed. This work is based on a predictive model which was proposed by the authors for orthogonal cutting. The thermo-mechanical effect is incorporated using the Johnson-Cook material model and a thermal model considering the tool thermal properties. An equivalent cutting edge is applied to include the cutting forces generated by the end cutting edge. The true tooth path and radial run-out are included in the chip thickness model. The proposed force model is verified under different cutting conditions. The results show that acceptable force predictions have been obtained in end milling of titanium alloy, which indicate a reasonable assumption of the semi-stationary serrated chip formation. The proposed force model with an equivalent cutting edge improves the force predictions in the $x$ and $y$ directions. 
Both the force models with and without the equivalent cutting edge predict the forces in the $z$ direction close to the measured ones. It is noted that the predicted forces in up milling differ from the experimental ones in terms of phase width. Future work is still needed to improve this model in up milling. The proposed model overcomes the drawbacks of the FE and mechanistic methods which require many simulations and experimental tests to determine the cutting force coefficients.

\section{References}

1. Chen Y, Li HZ, Wang J. Cutting Forces and Tool Wear in Dry Milling of Ti6Al4V. Adv Mat Res. 2012; 565: 454-9.

2. Peng C, Wang L, Li Z, Yang Y. Time-domain simulation and experimental verification of dynamic cutting forces and chatter stability for circular corner milling. P I Mech Eng B-J Eng. 2015; 229: 932-9.

3. Yoon MC, Chin DH. Cutting force monitoring in the endmilling operation for chatter detection. P I Mech Eng B-J Eng. 2005; 219: 455-65.

4. Kara F, Aslantas K, Cicek A. Prediction of cutting temperature in orthogonal machining of AISI 316L using artificial neural network. Appl Soft Comput. 2016; 38: 64-74.

5. Kline WA, DeVor RE. The effect of runout on cutting geometry and forces in end milling. Int J Mach Tool Manu. 1983; 23: 123-40.

6. Uriarte L, Azcárate S, Herrero A, Lopez de Lacalle LN, Lamikiz A. Mechanistic modelling of the micro end milling operation. P I Mech Eng B-J Eng. 2008; 222: 23-33.

7. Landers RG, Galecki G, Young K, Hanks R. Peripheral milling of thin titanium plates: Modelling, analysis, and process planningg. P I Mech Eng B-J Eng. 2011; 225: 783-98.

8. Yucesan G, Altintas Y. Improved modelling of cutting force coefficients in peripheral milling. Int J Mach Tools Manuf. 1994; 34: 473-87.

9. Lamikiz A, Lopez de Lacalle LN, Sanchez JA, Bravo U. Calculation of the specific cutting coefficients and geometrical aspects in sculptured surface machining. Mach Sci Technol. 2005; 9: 411-36.

10. Budak E, Armarego EJA, Altintas Y. Prediction of milling force coefficients from orthogonal cutting data. J Eng Ind Trans ASME. 1996; 118: 216-24. 
11. Dhanorker A, Ozel T. Meso/micro scale milling for micro-manufacturing. Int $J$ Mechatron Mech Syst. 2008; 1: 23-42.

12. Johnson GR, Cook WH. A constitutive model and data for metals subjected to large strains, high strain rates and high temperatures. Proceedings of the 7th International Symposium on Ballistics. Hague, Netherlands: P Int Sym Ball 1983, p. 541-7.

13. Gonzalo O, Jauregi H, Uriarte L, de Lacalle LL. Prediction of specific force coefficients from a FEM cutting model. Int J Adv Manuf Tech. 2009; 43: 348-56.

14. Wang ZG, Rahman M, Wong YS, Li XP. A hybrid cutting force model for high-speed milling of titanium alloys. CIRP Ann Manuf Technol. 2005; 54: 71-4.

15. Oxley PLB. The mechanics of machining: An analytical approach to assessing machinability. Chichester, England: Ellis Horwood Limited, 1989.

16. Matsumura $\mathrm{T}$, Tamura $\mathrm{S}$. Force prediction in milling of titanium alloy. ASME/ISCIE 2012 International Symposium on Flexible Automation. St. Louis, MO, United states 2012, p. 525-31.

17. Moufki A, Dudzinski D, Le Coz G. Prediction of cutting forces from an analytical model of oblique cutting, application to peripheral milling of Ti-6Al-4V alloy. Int $J A d v$ Manuf Technol. 2015; 81: 615-26.

18. Becze CE, Elbestawi MA. A chip formation based analytic force model for oblique cutting. Int J Mach Tools Manuf. 2002; 42: 529-38.

19. Li XP, Li HZ. Theoretical modelling of cutting forces in helical end milling with cutter runout. Int J Mech Sci. 2004; 46: 1399-414.

20. Manyindo BM, Oxley PLB. Modelling the catastrophic shear type of chip when machining stainless steel. P I Mech Eng C-J Mec. 1986; 200: 349-58.

21. Vyas A, Shaw MC. Mechanics of Saw-Tooth Chip Formation in Metal Cutting. J Manuf Sci Eng. 1999; 121: 163-72.

22. Chen Y, Li H, Wang J. Analytical modelling of cutting forces in near-orthogonal cutting of titanium alloy Ti6Al4V. P I Mech Eng C-J Mec. 2014; 229: 1122-33.

23. Lazoglu I, Altintas Y. Prediction of tool and chip temperature in continuous and interrupted machining. Int J Mach Tools Manuf. 2002; 42: 1011-22.

24. Hu RS, Mathew P, Oxley PLB, Young HT. Allowing for end cutting edge effects in predicting forces in bar turning with oblique machining conditions. P I Mech Eng C-J Mec. 1986; 200: 89-99. 
25. Lin GCI, Mathew P, Oxley PLB, Watson AR. Predicting cutting forces for oblique machining conditions. P I Mech Eng. 1982; 196: 141-8.

26. Stabler GV. The Fundamental Geometry of Cutting Tools. P I Mech Eng. 1951; 165: 1426.

27. Astakhov VP. Geometry of single-point turning tools and drills: fundamentals and practical applications: Springer Science \& Business Media, 2010.

28. Li HZ, Liu K, Li XP. A new method for determining the undeformed chip thickness in milling. J Mater Process Technol. 2001; 113: 378-84.

29. Press WH, Teukolsky SA, Vetterling WT, Flannery BP. Numerical Recipes: the art of scientific computing, Third Edition $(C++)$ : Cambridge University Press, 2007.

30. Lacalle LNLd, Lamikiz A, Sanchez JA, Bustos IFd. Simultaneous measurement of forces and machine tool position for diagnostic of machining tests. IEEE Trans Instrum Meas. 2005; 54: 2329-35.

31. Lacalle LNLd, Lamikiz A, Sánchez JA, Bustos IFd. Recording of real cutting forces along the milling of complex parts. Mechatronics. 2006; 16: 21-32.

32. Jin X, Altintas Y. Prediction of micro-milling forces with finite element method. $J$ Mater Process Technol. 2012; 212: 542-52.

33. Wan M, Zhang W-H, Yang Y. Phase width analysis of cutting forces considering bottom edge cutting and cutter runout calibration in flat end milling of titanium alloy. J Mater Process Technol. 2011; 211: 1852-63.

34. Schmitz TL, Smith KS. Machining dynamics: frequency response to improved productivity: Springer Science \& Business Media, 2008.

35. Wan M, Zhang WH, Qin GH, Tan G. Efficient calibration of instantaneous cutting force coefficients and runout parameters for general end mills. Int J Mach Tools Manuf. 2007; 47: 1767-76. 